\title{
Chemotherapy-induced cardiotoxicity: A major underestimated unmet medical need
}

\author{
Pierre A Guertin* \\ Department of Psychiatry and Neurosciences, Laval University Medical Center - CHU de Quebec, Canada
}

\begin{abstract}
Heart problems caused secondarily by anticancer drugs are increasingly being documented by epidemiologists. However, beyond the anthracycline story uncovered in the late 60 s, the cardiotoxicity level, the current situation and future challenges associated with chemotherapy-induced heart problems remains poorly characterized. For instance, the incidence of cancer-surviving patients who died of cardiac failure a few years post-chemotherapy/radiotherapy is still a subject of debate. Cardiac disease is already one of the most devastating causes of death among the population. This causal relationship between anticancer drugs and cardiac problem is thus likely to constitute a much greater problem than originally believed - perhaps to the extent of reaching epidemic proportions. Yet, as of today, hematologists and oncologists accepting to prescribe cardio-protective drugs during chemotherapy and/or radiotherapy are rare. This is probably attributable to poor evidence-based medicine, risks of increasing adverse events, and potential drug-drug interactions potentially reducing anticancer drug efficacy. Given the development of new therapies, increasing cancer patients of young age and increased survival time post-treatment, detection of cardiotoxic effects detected within months or years post-chemotherapy is bound to become one of the largest unmet medical needs. It is imperative for clinicians to begin systematically building publically-accessible databases of cancer survivors and related-heart problems in order for epidemiologists to conduct extensive studies and meta-analyses aimed at providing clear evidence about how, when and if prescription of cardio-protective drugs during chemotherapy should be authorized.
\end{abstract}

\section{Editorial}

According to the World Health Organization (WHO), 17.5 million people die each year from cardiovascular diseases - that is $31 \%$ of all deaths worldwide [1]. Cancer is also devastating with 8.2 million patients dying each year [2]. Unfortunately, new cases diagnosed with cancer are expected to rise dramatically by about $70 \%$ over the next twenty years [2]. Prevalence including both survivors and nonsurvivors shall then increase from 14 million patients up to 22 million cases - that is 13 million deaths directly attributable to cancer each year because of the expected aging population [3].

This said, more and more patients diagnosed with cancer become socalled survivors or cancer-free [4]. Unfortunately, increasing evidence from epidemiologists suggests that a significant proportion will, within months or years post-chemotherapy, die instead of cardiac disease caused by chemotherapy and/or radiotherapy. According to a few experts, this relatively new unmet medical need that is chemotherapyinduced heart disease will likely reach epidemic proportions given the increasing number of 'so-called' survivors and increasing prevalence of cancer due to aging and global population explosion $[5,6]$. As of now, about $50 \%$ of all cancer cases survive to the disease for at least ten years or more [7].

Anthracyclines are antibiotics derived from Streptomyces that have been widely used as chemotherapy for more than fifty years against different types of cancer including leukemias, lymphomas,

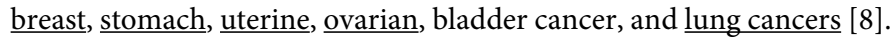
Daunorubicin, one of the first anthracyclines used as chemotherapy, was rapidly reported in the late $60 \mathrm{~s}$ and early 70 s to induce significant cardiac adverse events including cardiomyopathies and cardiac failures in children [9]. This said, a complete list of currently used chemotherapy agents capable of dose-dependently inducing cardiovascular disease remains lacking.

Still today, anthracyclines are considered among some of most efficacious anticancer agents ever invented. However, their dosedependent cardiotoxicity has now been clearly shown to be devastating [10]. For instance, in children, the incidence of asymptomatic myocardial dysfunction ranges from 18 to $57 \%$ among survivors post-oncological treatment whereas about $5 \%$ of them develop heart failure [11]. Many decades after diagnosis, survivors have 15-fold increased rates of congestive heart failure and 10-fold higher rates of cardiovascular disease compared with controls [12,13]. Cancerrelated cardiovascular morbidity, mortality and risks may persist up to 45 years after therapy [14].

However, this could be only the tip of the iceberg. Indeed, in recent years, additional anticancer drugs such as alkylating agents (cyclophosphamide, ifosfamide), platinum agents, antimetabolites (5-fluorouracil, capecitabine), antibiotics (mitoxantrone, mitomycin, bleomycin), and antimicrotubule agents (taxanes) were found to cause heart disease [15]. Even if physical activity and at least one drug, dexrazoxane, have been shown to prevent and mitigate cardiotoxicity related to anthracycline therapy [16], the latter has been reported shortly after approval to increase also risks of acute leukemia and myelodysplastic syndrome according to the FDA [17].

Given that $5 \%$ and between $18 \%$ and $57 \%$ of cancer survivors

Correspondence to: Pierre A Guertin, Department of Psychiatry and Neurosciences, Laval University Medical Center - CHU de Quebec, Canada, E-mail: pierre.guertin@crchul.ulaval.ca

Received: February 14, 2017; Accepted: February 21, 2017; Published: February 22, 2017 
experience heart failure and asymptomatic cardiac dysfunction respectively [11], then by 2035 , if no safe cardio-protective agents has been approved, 0.6 million cancer survivors will die of heart failure and between 2 and 7 million patients will develop asymptomatic myocardial dysfunction within a few months to a few years post-cancer treatments. That is eight times greater than the number of Americans currently living with Parkinson's disease [18].

In other words, cardiotoxicity induced by cancer treatments should be considered by pharmaceutical companies has an unmet medical need indication with an increasing rapidly growing market. As such, corresponding efforts should be invested in identifying, among all types of cardio-protective agents, the one(s) that can reduce cardiovascular complications without affecting the efficacy of commonly-used chemotherapeutic agents against cancer.

\section{References}

1. 22 September, 2016: "Global Hearts", a new initiative from the World Health Organization (WHO). New initiative launched to tackle cardiovascular disease, the world's number one killer. Cardiovascular disease. http://www.who.int/cardiovascular_ diseases/en/

2. WHO Media centre (2017) Cancer. http://www.who.int/mediacentre/factsheets/fs297/en/

3. Global Cancer Facts \& Figures. American cancer society. https://www.cancer.org/ research/cancer-facts-statistics/global.html

4. Lucas G, Marcu A, Piano M, Grosvenor W, Mold F, et al. (2017) Cancer suvivors's experience with telehealth: a systematic review and thematic synthesis. J Med Internet Res 19: e11.

5. Thavendiranathan P, Nolan MT (2016) An emerging epidemic: cancer and heart failure. Clinical Science 131 (2) 113-121.
6. White MC, Holman DM, Boehm JE, Peipins LA, Grossman M, et al. (2015) Age and cancer risk - a potentially modifiable relationship. Am J Prev Med 46: 7-15. http:// www.cancerresearchuk.org/

7. Fujiwara A, Hoshino T, Westley JM (1985) Anthracycline antibiotics. Critical Reviews in Biotechnology 3: 133-157.

8. Laine JL, Julienne O, Leroy J, Lamagnère JP, Laugier J, et al. (1973) Cardiac toxicity of rubidomycin. Apropos of two cases. Pédiatrie (French) 28: 201-210.

9. Mele D, Nardozza M, Spallarossa P, Frassoldati A, Tocchetti CG, et al. (2016) Current views on anthracycline cardiotoxicity. Heart Fail Rev 21: 621-634.

10. Kucharska W, Negrusz-kawecka M, Gromkowska M (2012) Cardiotoxicity of oncological treatment in children. Adv Clin Exp Med 21: 281-218.

11. Menna P, Salvatorelli E, Minotti G (2008) Cardiotoxicity of antitumor drugs. Chem Res Toxicol 21: 978-989.

12. Cardinale D, Bacchiani G, Beggiato M, et al (2013) Strategies to prevent and treat cardiovascular risk in cancer patients. Semin Oncol 40: 186-198.

13. Reulen RC, Winter DL, Frobisher C, et al (2010) Long-term cause-specific mortality among survivors of childhood cancer. JAMA 304: 172-179.

14. Madeddu C, Deidda M, Piras A, Cadeddu C, Demurtas L, et al. (2016) Pathophysiology of cardiotoxicity induced by nonanthracycline chemotherapy. J Cardiovascu Med 17: $12-18$

15. Chen JJ, Wu PT, Middlekauff HR, Nguyen KL (2016) Aerobic Exercise in Anthracycline-Induced Cardiotoxicity: A Systematic Review of Current Evidence and Future Directions. Am J Physiol Heart Circ Physiol 2016: Dec 6: ahead of print.

16. United States Food and Drug Administration (2011) FDA Statement on Dexrazoxane. Drugs. http://www.fda.gov/Drugs/DrugSafety/ucm263729.htm

17. Capriotti T, Terzakis K (2016) Parkinson disease. Home Health Now 34: 300-307.

Copyright: $(2017$ Guertin PA. This is an open-access article distributed under the terms of the Creative Commons Attribution License, which permits unrestricted use, distribution, and reproduction in any medium, provided the original author and source are credited. 